Check for updates

Cite this: RSC Adv., 2017, 7, 36550

Received 18th June 2017

Accepted 18th July 2017

DOI: $10.1039 / c 7 r a 06777 a$

rsc.li/rsc-advances

\title{
Why is sodium-intercalated graphite unstable?
}

\author{
Hiroki Moriwake, (D)* Akihide Kuwabara, Craig A. J. Fisher and Yuichi Ikuhara
}

$\mathrm{Na}$-ion batteries offer an attractive low-cost alternative to Li-ion batteries. Although graphite is used as the negative electrode in conventional Li-ion batteries, attempts to use it in $\mathrm{Na}$-ion batteries have been hampered by the inability of $\mathrm{Na}$ to form graphite intercalation compounds (GICS) under moderate conditions. It is generally considered that this is due to the size mismatch between $\mathrm{Na}$ and the graphite interlayer spacings, but here we show with detailed first-principles calculations of $\mathrm{Li}, \mathrm{Na}, \mathrm{K}, \mathrm{Rb}$, and $\mathrm{Cs}$ GICs that the major reason is the change in chemical bonding between alkali metal (AM) ion and $C$ atoms. van der Waals correction terms are introduced to better reproduce the layered graphite structure, and calculated formation energies of $\mathrm{GICs} A M C_{6}$, where $A M=\mathrm{Li}, \mathrm{Na}, \mathrm{K}, \mathrm{Rb}$, and $\mathrm{Cs}$, are found to become less negative (less stable) as ion size decreases from $\mathrm{Cs}$ to $\mathrm{Na}$ as a result of weakening ionic bonding, until the formation energy of $\mathrm{NaC}_{6}$ becomes positive. The much smaller $\mathrm{Li}$ ion represents an exception to this trend, as its bonds with $\mathrm{C}$ atoms contain a covalent component, resulting in a negative formation energy. These subtle differences in staging for the different alkali metals explain why graphite is a good intercalation material for $\mathrm{Li}$ and $\mathrm{K}$ but not for $\mathrm{Na}$.

\section{Introduction}

As demand for sustainable energy sources continues to grow, so does the need for cost-effective, high-efficiency, environmentally benign, and durable energy storage devices. Lithium-ion batteries (LIBs), widely used as power sources in mobile electronics (e.g., cell phones, laptops, and PCs) because of their high energy densities and good durability, are today being scaled up or connected in series to power electric vehicles, as well as serving as energy storage systems for intermittent power generators such as wind turbines and solar panels. For these large-scale applications, however, limited lithium reserves are a serious concern, and in recent years efforts to develop practical sodium-ion batteries (SIBs) have increased rapidly because of sodium's much greater crustal abundance. ${ }^{1-6}$ Compared to the range of sodium-intercalating materials proposed for use as positive electrodes, ${ }^{1-8}$ the materials known to be suitable for use as the negative electrode are considerably fewer in number. ${ }^{1-6,9}$

In conventional LIBs, graphite is commonly used as the negative electrode because of its ability to reversibly intercalate $\mathrm{Li}$ ions, forming a series of binary graphite intercalation compounds (GICs) as it does so. With the notable exception of $\mathrm{Na}$, the other alkali metals (AMs) are also known to form stable GICs. ${ }^{10}$

Graphite is a layered material with sp2 hybridized carboncarbon bonds within the graphene layers and weak van der Waals (vdW) interactions between the graphene layers. Graphite intercalated with electron donors such as alkali metals display a rich variety of phases with different compositions and crystal

Nanostructures Research Laboratory, Japan Fine Ceramics Center, 2-4-1 Mutsuno, Atsuta-ku, Nagoya, 456-8587 Japan structures. $\mathrm{LiC}_{6}$ and $\mathrm{KC}_{8}$ are examples of stage-1 GICs with the metal intercalated between all the graphite layers. The staging index refers to the number of graphene layers between two successive layers of intercalated alkali metal atoms. A low stage index Na-GIC has never been observed; only higher stage compounds such as $\mathrm{NaC}_{48}, \mathrm{NaC}_{64}, \mathrm{NaC}_{80}$, in which some $\mathrm{Na}$ is intercalated in every eighth layer or less, have ever been reported. ${ }^{11-13}$

Recent theoretical studies by Nobuhara et al. ${ }^{14}$ Wang et al. ${ }^{15}$ Okamoto, ${ }^{16}$ and Liu et al. ${ }^{17}$ using vdW functionals reported similar results. Both confirmed that lower-stage Na-GICs are unstable. Strategies to overcome this limitation based on the formation of ternary GICs have been proposed, for example, the intercalation of solvated $\mathrm{Na}$ ions ("co-intercalation"). ${ }^{18,19}$ Wen et al. ${ }^{20}$ have also reported that expanded graphite, in which the spacing between layers is increased by introducing functional groups, accepts a larger amount of $\mathrm{Na}$ intercalation, and the intercalation process was examined using first-principles calculations by Kang et $a .^{21}$ In the case of graphite under normal conditions, however, the underlying mechanisms inhibiting $\mathrm{Na}$ intercalation remain poorly understood. To uncover the underlying reasons why only $\mathrm{Na}$ out of the alkali metals cannot be intercalated into graphite, we have carried out systematic first-principles calculations of the five AM-GIC systems using a vdW functional to reproduce the correct structure of the graphite crystal.

\section{Methodology}

First-principles calculations were performed using the projector-augmented wave (PAW) method $^{22}$ within the 
framework of density functional theory (DFT), ${ }^{23}$ as implemented in the VASP code. ${ }^{\mathbf{2 4 5}}$ Exchange-correlation interactions were treated by the generalized gradient approximation (GGA-PBE) ${ }^{26}$ with five different van der Waals (vdW) functionals, ${ }^{27,28}$ namely vdW-revPBE, ${ }^{27}$ vdW-optPBE, ${ }^{29}$ vdW-optB88, ${ }^{29}$ vdW-optB86b, ${ }^{30}$ and vdW-D2, ${ }^{31}$ implemented using the algorithm of RománPérez and Soler. ${ }^{32}$

In the case of vdW-optB88, vdW-optB86b, and vdW-D2 functionals, default values were used for the input parameters. Calculations using standard functionals GGA-PBE and GGA-PBE_sol ${ }^{33}$ were also carried out for comparison. For the PAW potentials, $1 \mathrm{~s}$ and $2 \mathrm{~s}$ for $\mathrm{La}, 2 \mathrm{p}$ and $3 \mathrm{~s}$ for $\mathrm{Na}, 3 \mathrm{p}$ and $4 \mathrm{~s}$ for $\mathrm{K}, 4 \mathrm{p}$ and $5 \mathrm{~s}$ for $\mathrm{Rb}, 5 \mathrm{p}$ and $6 \mathrm{~s}$ for $\mathrm{Cs}$, and $2 \mathrm{~s}$ and $2 \mathrm{p}$ for $\mathrm{C}$ were explicitly treated as valence electrons. Planewave expansion was performed up to $500 \mathrm{eV}$ with $k$-point spacings of the Brillouin zone of $0.25 \mathrm{~A}^{-1}$ using a $\Gamma$-point centered Monkhorst-Pack scheme. ${ }^{34}$ Lattice constants and internal atomic coordinates were considered fully optimized when residual HellmannFeynman forces were smaller than $10^{-3} \mathrm{eV} \AA^{-1}$.

The stabilities of AM-GICs were evaluated by calculating their formation energies $(\Delta E)$ according to

$$
\Delta E=E_{\mathrm{AM}-\mathrm{GIC}}-E_{\text {graphite }}-E_{\mathrm{AM}}
$$

where $E_{\mathrm{AM}-\mathrm{GIC}}, E_{\text {graphite }}$, and $E_{\mathrm{AM}}$ are the total energies of the AMGIC, pure graphite, and alkali metal, respectively, after structure optimization.

\section{Results and discussion}

\section{Comparison of van der Waals functionals}

Previous studies have shown the importance of van der Waal's forces in holding the $2 \mathrm{D}$ graphite structure together. ${ }^{14-17,35}$ It is also well known that the conventional GGA functional is unable to reproduce the layered structure of graphite, while the LDA functional can. ${ }^{\mathbf{1 5}, 36}$ Neither functional explicitly includes transient polar interactions, so the different results likely stem from the fact the LDA functional overestimates binding between atoms. Recently, many types of functionals have been developed to reproduce dispersive van der Waals interactions in weakly bonded systems. In this study, five types of van der Waals functionals were tested to assess how well they reproduce the structures and energetics of pure graphite and AM-GICs. Structural parameters obtained from the different functionals are compared in Tables 1 and 2 for pure graphite and $\mathrm{LiC}_{6}$, respectively. Most functionals apart from conventional GGA predict the graphite structure to be stable, but the vdW-D2 functional underestimates the $c$ parameter by over $4 \%$, while the GGA-PBEsol and GGA + vdW-revPBE functionals overestimate it by more than $5 \%$. In contrast, in the case of $\mathrm{LiC}_{6}$, all functionals, including the conventional GGA-PBE and GGAPBE_sol functionals, reproduced the structure reasonably well, consistent with previous studies. ${ }^{\mathbf{1 4 - 1 7}}$ This is because the intercalated $\mathrm{Li}$ atoms bond with $\mathrm{C}$ to hold the graphene sheets together. Of the functionals examined, the vdW-optPBE functional produced the smallest deviations from experiment for
Table 1 Lattice parameters of graphite calculated using different van der Waals functionals

\begin{tabular}{llll}
\hline Functional & \multicolumn{2}{l}{ Parameter $(\AA)$} & Deviation $^{a}(\%)$ \\
\hline \multirow{2}{*}{ GGA-PBE } & $a$ & - & - \\
GGA-PBEsol & $c$ & - & - \\
& $a$ & 2.461 & -0.11 \\
vdW-D2 & $c$ & 7.063 & +5.25 \\
& $a$ & 2.464 & -0.01 \\
vdW-optB86b & $c$ & 6.417 & -4.38 \\
& $a$ & 2.468 & +0.15 \\
vdW-optB88 & $c$ & 6.601 & -1.64 \\
& $a$ & 2.466 & +0.10 \\
vdW-optPBE & $c$ & 6.695 & -0.24 \\
& $a$ & 2.473 & +0.35 \\
vdW-revPBE & $c$ & 6.841 & +1.93 \\
& $a$ & 2.479 & +0.60 \\
$a$ & $c$ & 7.095 & +5.72
\end{tabular}

Table 2 Calculated lattice parameters of $\mathrm{LiC}_{6}$

\begin{tabular}{|c|c|c|c|}
\hline \multirow{2}{*}{$\begin{array}{l}\text { Functional } \\
\text { GGA-PBE }\end{array}$} & \multicolumn{2}{|c|}{ Parameter $(\AA)$} & \multirow{2}{*}{$\begin{array}{l}\text { Deviation }^{a}(\%) \\
+0.25\end{array}$} \\
\hline & $a$ & 4.327 & \\
\hline & $c$ & 3.761 & +1.64 \\
\hline \multirow[t]{2}{*}{ GGA-PBEsol } & $a$ & 4.314 & -0.06 \\
\hline & $c$ & 3.683 & -0.46 \\
\hline \multirow[t]{2}{*}{ vdW-D2 } & $a$ & 4.321 & +0.12 \\
\hline & $c$ & 3.655 & -1.22 \\
\hline \multirow[t]{2}{*}{ vdW-optB86b } & $a$ & 4.327 & +0.24 \\
\hline & $c$ & 3.636 & -1.72 \\
\hline \multirow[t]{2}{*}{ vdW-optB88 } & $a$ & 4.325 & +0.21 \\
\hline & $c$ & 3.648 & -1.39 \\
\hline \multirow[t]{2}{*}{ vdW-optPBE } & $a$ & 4.336 & +0.45 \\
\hline & $c$ & 3.684 & -0.43 \\
\hline \multirow[t]{2}{*}{ vdW-revPBE } & $a$ & 4.348 & +0.73 \\
\hline & $c$ & 3.756 & +1.52 \\
\hline
\end{tabular}

both the graphite and $\mathrm{LiC}_{6}$ structures, so this was chosen for further calculations.

\section{Stability of alkali-metal graphite intercalation compounds}

Formation energies were calculated for different alkali metal concentrations in the graphite layers. Similar to previous reports, ${ }^{\mathbf{1 4}, \mathbf{1 5}}$ energy differences between different stacking sequences of the graphite layers for the same alkali metal content were relatively small, so for the purpose of comparison, AA stacking of graphite sheets was assumed hereafter. Using the above assumptions, formation energies of the $\mathrm{AMC}_{6}$ series $\left(\mathrm{AMC}_{6}, \mathrm{AMC}_{12}, \mathrm{AMC}_{24}, \mathrm{AMC}_{36}, \mathrm{AMC}_{48}, \mathrm{AMC}_{72}\right)$ and $\mathrm{AMC}_{8}$ series $\left(\mathrm{AMC}_{8}, \mathrm{AMC}_{16}, \mathrm{AMC}_{32}, \mathrm{AMC}_{64}, \mathrm{AMC}_{80}\right)$ for $\mathrm{AM}=\mathrm{Li}, \mathrm{Na}$, and $\mathrm{K}$ were calculated. The results for $\mathrm{Li}-\mathrm{C}, \mathrm{K}-\mathrm{C}$, and $\mathrm{Na}-\mathrm{C}$ systems are summarized in Fig. 1(a)-(c), respectively. In Fig. 1(a), LiGICs are stable even for low-stage structures (high Li concentrations). In fact, the stability increases with increasing Li 
(a)

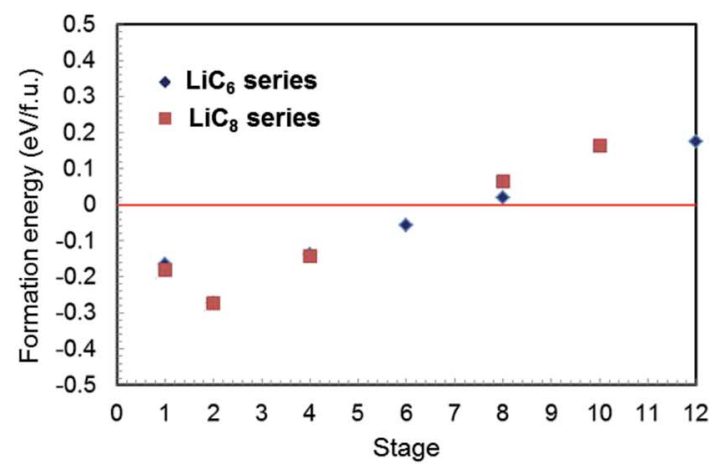

(b)

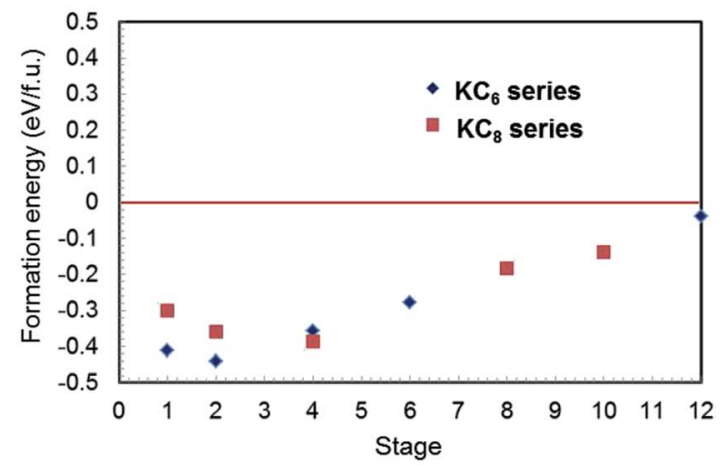

(c)

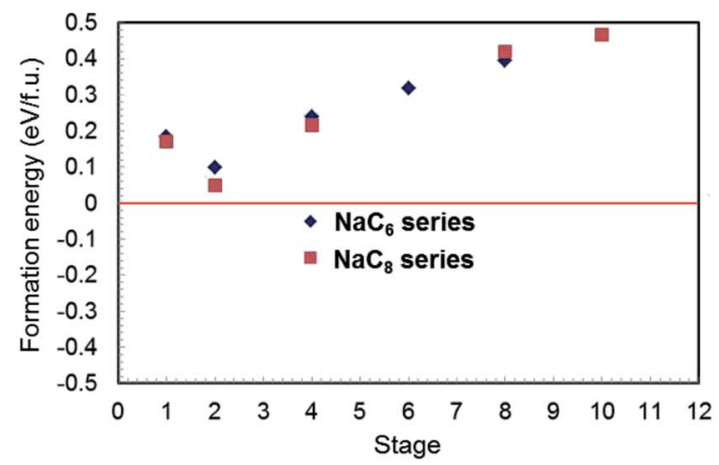

Fig. 1 Calculated formation energies of $A M-G I C s(A M=L i, N a$ and $K)$ as a function of stage number for $A M C_{6}$ and $A M C_{8}$ series: (a) Li-GIC, (b) $\mathrm{K}-\mathrm{GIC}$ and (c) $\mathrm{Na}-\mathrm{GIC}$

content down to stage 2 , before decreasing slightly for stage 1 for both $\mathrm{LiC}_{6}$ and $\mathrm{LiC}_{8}$ series. As shown in Fig. 1(b), despite the large ionic radius of $\mathrm{K}, \mathrm{K}-\mathrm{GIC}$ is more stable than Li-GIC for the same stage and series, and unlike the Li-GIC system, K-GICs are stable over the entire range of stages considered. Perhaps surprisingly, the change in stabilities with staging level follows the same trend as for Li-GICs. In contrast, Fig. 1(c) shows that the formation energies of Na-GICs are positive over the entire range of stages examined, indicating that Na-GIC is thermodynamically unstable for all compositions. Apart from this, however, the same trend is followed as for Li-GICs and K-GICs. These trends are in good agreement not only with previous experimental results ${ }^{11-13}$ but with theoretical reports ${ }^{14-16}$ as well.

As expressed by eqn (1), the formation energies of AM-GICs are a function of the total energy of the intercalated compound, the total energy of graphite, and the total energy of the pure metal. When comparing the different systems, the total energy of graphite is constant, so the trends in AM-GIC formation energies must be due to changes in the stabilities of the alkali metals or changes in the intercalated system energies. It would be easy to rationalize the instability of Na-GICs if $\mathrm{Na}$ metal were exceptionally stable, for example. However, of these three alkaline metals, Li metal is most stable (lowest energy), decreasing as the atomic radius increases. The reason for the deviation in linearity for the formation energies must lie in the nature of the AM-GICs themselves.

\section{Origin of the instability of Na-GICs}

Of the AM-GICs examined in the previous section, only Na-GIC is unstable for all intercalation stages. Extending these calculations to a wider range of alkali metals suggests that it is actually Li-GICs that deviate from the trend rather than Na-GIC. For example, Fig. 2 shows calculated formation energies of $\mathrm{AMC}_{6}$ structures where $\mathrm{AM}=\mathrm{Li}, \mathrm{Na}, \mathrm{K}, \mathrm{Rb}$ and Cs. Of the five alkali metals, only $\mathrm{NaC}_{6}$ has a positive formation energy. For cations larger than $\mathrm{Na}^{+}$, the formation energy becomes more negative (more stable) as the ion radius increases.

The electronegativity of alkali metal atoms decreases down the period, meaning that the larger the ion radius, the greater the iconicity of the bond between metal and $\mathrm{C}$ atoms. Conversely, as the ion radius decreases, the metal-carbon bond becomes progressively weaker, with formation energies becoming more positive until for Na-GIC the formation energy is positive (i.e., $\mathrm{NaC}_{6}$ is unstable). For the smallest alkali metal, $\mathrm{Li}$, the formation energy of the intercalated compound breaks from this trend, becoming negative again, consistent with LiGICs being observed to be stable by experiment.

The reason for this apparent anomaly is that the binding energies of the GICs consist not only of an ionic component but also of a covalent component between the metal and carbon atoms. ${ }^{36}$ Maps of electron densities through the centers of AM and $\mathrm{C}$ atoms in $\mathrm{AMC}_{6}$ compounds for $\mathrm{AM}=\mathrm{Li}, \mathrm{Na}$, and $\mathrm{K}$, plotted to the same scale, are shown in Fig. 3. In the case of $\mathrm{KC}_{6}$ and $\mathrm{NaC}_{6}$, the electron density between $\mathrm{C}$ and $\mathrm{AM}$ is negligibly small. In the case of $\mathrm{LiC}_{6}$, the bond length is shorter and the electron density between $\mathrm{C}$ and $\mathrm{Li}$ atoms is greater. This indicates that in Li-C bonds there is a certain amount of sharing of electrons between atom species, in other words, a nonnegligible covalent bond component. In contrast to Na-GIC and K-GIC, the increased covalent bonding in Li-GICs is sufficiently large to overcome the decrease in the ionic component,

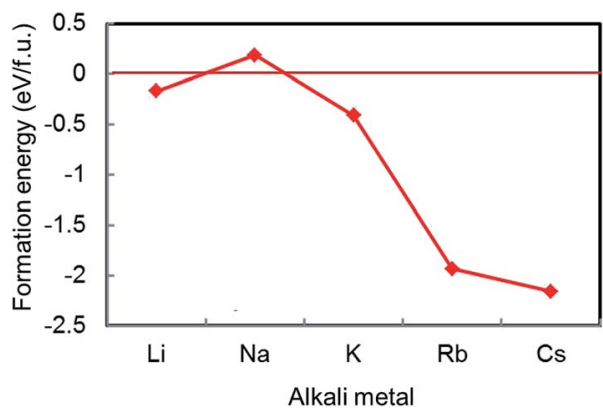

Fig. 2 Calculated formation energies of $A M C_{6}$ for $A M=L i, N a, K, R b$, and $\mathrm{Cs}$ in order of increasing atomic number. 


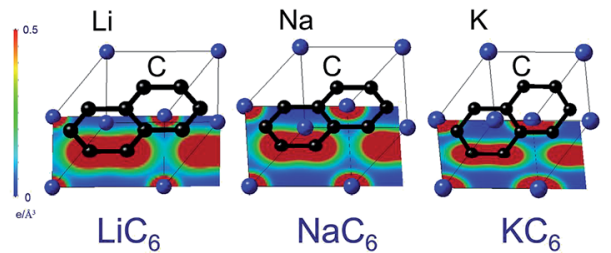

Fig. 3 Electron densities for compositions $\mathrm{LiC}_{6}, \mathrm{NaC}_{6}$ and $\mathrm{KC}_{6}$ plotted to the same scale. For $\mathrm{KC}_{6}$ and $\mathrm{NaC}_{6}$, the electron density between $\mathrm{C}$ and $\mathrm{AM}$ atoms is negligibly small. For $\mathrm{LiC}_{6}$, in contrast, the electron density between $\mathrm{C}$ and $\mathrm{Li}$ is greater, indicating some covalency in the bonding between $\mathrm{C}$ and $\mathrm{Li}$. This covalent contribution stabilizes $\mathrm{Li}-\mathrm{GIC}$ layers, despite the small size of the Li atom.

so that Li-GICs are more stable than Na-GICs. In other words, it is the balance between the proportion of iconicity and covalency in the metal-carbon bond that determines the shape of the formation energy versus ionic radius plot. Without the contribution from covalent bonding between $\mathrm{C}$ and $\mathrm{Li}$ atoms, Li-GICs would be even less stable than Na-GICs.

\section{Conclusions}

Density functional theory calculations incorporating van der Waals functionals to reproduce accurately the graphite layer structure were performed to evaluate the stabilities of alkalimetal graphite intercalation compounds. The main findings can be summarized as follows: Na-GIC was found to be thermodynamically unstable for all $\mathrm{Na}$ concentrations examined (stages 1 to 12). Li-, Na-, and K-GICs exhibit similar changes in formation energy as a function of stage number, although the absolute energies are very different. Of the wide range of staged structures examined, stage-2 AM-GICs, rather than stage-1 AMGICs, were found to be the most stable (lowest energy) for $\mathrm{Li}$, $\mathrm{Na}$, and K. Of the five alkali metals examined, only Na-GICs were found to be unstable for all intercalation stages. Comparison of the formation energies of stage-1 AM-GICs revealed that $\mathrm{Na}$ actually conforms to the expected intercalation behavior extrapolated from the behavior of the larger alkali metals. Rather than $\mathrm{Na}$, it is $\mathrm{Li}$ that is the exception to the trend obeyed by the alkali metals because, unlike the larger alkali metals, LiC bonds contain a non-negligible covalent component that helps stabilize the intercalated layered material.

\section{Acknowledgements}

This work was partially supported by the Research and Development Initiative for Scientific Innovation of New Generation Batteries II (RISING II) project from the New Energy and Industrial Technology Development Organization (NEDO), Japan. The authors acknowledge to Dr Masafumi Nose, Dr Hideki Nakayama, Dr Shinji Nakanishi, Dr Hideki Iba (Toyota Motor Corporation), for their fruitful discussion and support.

\section{References}

1 M. D. Slater, D. Kim, E. Lee and C. S. Johnson, Adv. Funct. Mater., 2013, 23, 947-958.
2 V. Palomares, M. Casas-Cabanas, E. Castillo-Martínez, M. H. Han and T. Rojo, Energy Environ. Sci., 2013, 6, 23122337.

3 H. Pan, Y.-S. Hu and L. Chen, Energy Environ. Sci., 2013, 6, 2338-2360.

4 N. Yabuuchi, K. Kubota, M. Dahbi and S. Komaba, Chem. Rev., 2014, 114, 11636-11682.

5 S.-W. Kim, D.-H. Seo, X. Ma, G. Ceder and K. Kang, Adv. Energy Mater., 2012, 2, 710-721.

6 L. P. Wang, L. Yu, X. Wang, M. Srinivasan and Z. J. Xu, J. Mater. Chem. A, 2015, 3, 9353-9378.

7 M. H. Han, E. Gonzalo, G. Singh and T. Rojo, Energy Environ. Sci., 2015, 8, 81-102.

8 X. Liu, X. Wang, A. Iyo, H. Yu, D. Lia and H. Zhou, J. Mater. Chem. A, 2014, 2, 14822-14826.

9 G. Hasegawa, K. Kanamori, T. Kiyomura, H. Kurata, K. Nakanishi and T. Abe, Adv. Energy Mater., 2015, 5, 1400730.

10 M. S. Drusselhaus and G. Dresselhaus, Adv. Phys., 2002, 51, 1-186.

11 A. Metrot, D. Guerard, D. Billaud and A. Herold, Synth. Met., 1979/80, 1, 363-369.

12 N. Adhouma, J. Bouteillon, D. Dumas and J. C. Poignet, Electrochim. Acta, 2006, 51, 5402-5406.

13 P. Ge and M. Fouletier, Solid State Ionics, 1988, 28-30, 11721175.

14 K. Nobuhara, H. Nakayama, M. Nose, S. Nakanishi and H. Iba, J. Power Sources, 2013, 243, 585-587.

15 Z. Wang, S. M. Selbach and T. Grande, RSC Adv., 2014, 4, 4069-4079.

16 Y. Okamoto, J. Phys. Chem. C, 2014, 118, 16-19.

17 Y. Liu, B. V. Merinova and W. A. Goddard III, Proc. Natl. Acad. Sci. U. S. A., 2016, 113, 3735-3739.

18 B. Jache and P. Adelhelm, Angew. Chem., Int. Ed., 2014, 53, 10169-10173.

19 G. Yoon, H. Kim, I. Park and K. Kang, Adv. Energy Mater., 2017, 7, 1601519.

20 Y. Wen, K. He, Y. Zhu, F. Han, Y. Xu, I. Matsuda, Y. Ishii, J. Cumings and C. Wang, Nat. Commun., 2014, 5, 4033.

21 Y.-J. Kang, S. C. Jung, J. W. Choi and Y.-K. Han, Chem. Mater., 2015, 27, 5402-5406.

22 P. E. Blöchl, Phys. Rev. B: Condens. Matter Mater. Phys., 1994, 50, 17953-17975.

23 W. Kohn and L. J. Sham, Phys. Rev., 1965, 140, A1133-A1138.

24 G. Kresse and J. Furthmüller, Phys. Rev. B: Condens. Matter Mater. Phys., 1996, 54, 11169-11186.

25 G. Kresse and D. Joubert, Phys. Rev. B: Condens. Matter Mater. Phys., 1999, 59, 1758-1775.

26 J. P. Perdew, K. Burke and M. Ernzerhof, Phys. Rev. Lett., 1996, 77, 3865-3868.

27 M. Dion, H. Rydberg, E. Schröder, D. C. Langreth and B. I. Lundqvist, Phys. Rev. Lett., 2004, 92, 246401.

28 T. Thonhauser, V. R. Cooper, L. Shen, A. Puzder, P. Hyldgaard and D. C. Langreth, Phys. Rev. B: Condens. Matter Mater. Phys., 2007, 76, 125112.

29 J. Klimeš, D. R. Bowler and A. Michaelides, J. Phys.: Condens. Matter, 2010, 22, 022201. 
30 J. Klimě̌, D. R. Bowler and A. Michaelides, Phys. Rev. B: Condens. Matter Mater. Phys., 2011, 83, 195131.

31 S. Grimme, J. Comput. Chem., 2006, 27, 1787-1799.

32 G. Román-Pérez and J. M. Soler, Phys. Rev. Lett., 2009, 103, 096102.

33 J. P. Perdew, A. Ruzsinszky, G. I. Csonka, O. A. Vydrov, G. E. Scuseria, L. A. Constantin, X. Zhou and K. Burke, Phys. Rev. Lett., 2008, 100, 136406.
34 H. J. Monkhorst and J. D. Pack, Phys. Rev. B: Solid State, 1976, 13, 5188-5192.

35 Y. Ma, Phys. Rev. B: Condens. Matter Mater. Phys., 2007, 76, 075419.

36 C. Hartwigsen, W. Witschel and E. Spohr, Phys. Rev. B: Condens. Matter Mater. Phys., 1997, 55, 4953-4959.

37 P. Trucano and R. Chen, Nature, 1975, 258, 136-137.

38 O. Dolotkoa, A. Senyshyn, M. J. Mühlbauer, K. Nikolowski and H. Ehrenberg, J. Power Sources, 2014, 255, 197-203. 